\title{
CORRIGENDUM
}

\section{Using milk leukocyte differentials for diagnosis of subclinical bovine mastitis - CORRIGENDUM}

Juliano Leonel Gonçalves, Roberta L. Lyman, Mitchell Hockett, Rudy Rodriguez, Marcos Veiga dos Santos and Kevin L. Anderson

doi:10.1017/S0022029917000267, Published online by Cambridge University Press, 27 June 2017

The authors would like to apologise for a mistake in the above-mentioned article by Gonçalves et al. Checking the data, it was found that the position of false-positive and false-negative results were accidently reversed when calculating sensitivity and specificity.

The following errors have been noted:

On the first page, in the abstract:

'When MC was considered the gold standard for mastitis diagnosis, the calculated diagnostic Se of the MLD was $65 \cdot 4 \%$ $\left(\mathrm{IC}_{95 \%}=57 \cdot 4\right.$ to $\left.72 \cdot 8 \%\right)$ and the $S p$ was $79 \cdot 3 \%\left(\mathrm{IC}_{95 \%}=71 \cdot 4\right.$ to $\left.85 \cdot 7 \%\right)$.'

Should read:

'When MC was considered the gold standard for mastitis diagnosis, the calculated diagnostic Se of the MLD was $78 \cdot 5 \%$ $\left(\mathrm{IC}_{95 \%}=70 \cdot 4\right.$ to $\left.85 \cdot 2 \%\right)$ and the $S p$ was $66 \cdot 5 \%\left(\mathrm{IC}_{95 \%}=58 \cdot 6\right.$ to $\left.73 \cdot 7 \%\right)$.'

On page 313, in the second column:

'When MC was considered the gold standard for mastitis diagnosis, the calculated diagnostic Se of the MLD was $65 \cdot 4 \%$ $\left(\mathrm{IC}_{95 \%}=57 \cdot 4\right.$ to $\left.72 \cdot 8 \%\right)$ and the $S p$ was $79 \cdot 3 \%\left(\mathrm{IC}_{95 \%}=71 \cdot 4 \%\right.$ to $\left.85 \cdot 7 \%\right)$. Using MC results as the 'gold standard,' Se and $S p$ of the categorical instrument readout results (healthy or infected) based upon cut-offs ranging from 1-12 are shown in Fig. 2. Sensitivity progressively increased from a minimum of $50 \cdot 4 \%$ at a user setting of 1 to a maximum of $71 \cdot 3 \%$ at a setting of 12 (Fig. 2). Specificity progressively decreased from a maximum of $86 \cdot 7 \%$ at user setting 1 to $66 \cdot 7 \%$ at setting 12 (Fig. 2).'

Should read:

'When MC was considered the gold standard for mastitis diagnosis, the calculated diagnostic Se of the MLD was $78 \cdot 5 \%$ $\left(\mathrm{IC}_{95 \%}=70 \cdot 4\right.$ to $\left.85 \cdot 2 \%\right)$ and the $S p$ was $66 \cdot 5 \%\left(\mathrm{IC}_{95 \%}=58 \cdot 6\right.$ to $\left.73 \cdot 7 \%\right)$. Using MC results as the 'gold standard,' Se and $S p$ of the categorical instrument readout results (healthy or infected) based upon cut-offs ranging from 1-12 are shown in Fig. 2. Sensitivity progressively decreased from a maximum of $95 \cdot 4 \%$ at a user setting of 1 to a minimum of $47 \cdot 7 \%$ at a setting of 12 (Fig. 2). Specificity progressively increased from a minimum of $24 \cdot 2 \%$ at user setting 1 to $84 \cdot 5 \%$ at setting 12 (Fig. 2),

On page 314, In Figure 2, the sensitivity and specificity results were accidently reversed, since the position of false-positive and false-negative results were mistakenly allocated in the $2 \times 2$ contingency table used for calculations.

The following is a corrected version of Figure 2: 


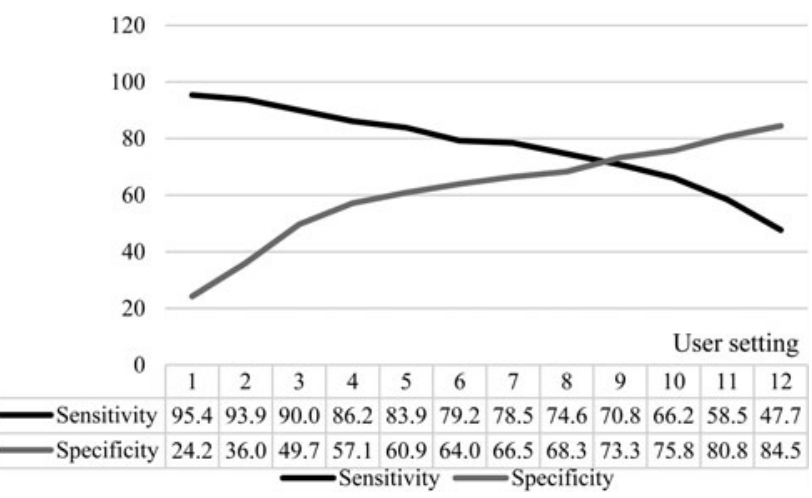

Fig. 2. Sensitivity and specificity evaluated at different threshold setting of QScout ${ }^{\circledR}$ MLD.

On page 316, in the 'Discussion', top of the right column:

'Our reported Se of $65.4 \%$ and $S p$ of $79 \cdot 3 \%$ were similar to those reported in prior studies. Pilla et al. (2013) reported Se of $73.3 \%$ and $S p$ of $73 \cdot 6 \%$. Adjustment of user settings from 1 to 12 would allow user optimization of settings. Sensitivities progressively increased from $50 \cdot 4 \%$ at setting 1 to $71 \cdot 3 \%$ at setting 12 , while specificities decreased from $86 \cdot 7 \%$ at setting 1 to $66 \cdot 7 \%$ at setting 12 (Fig. 2).'

Should read:

Our reported $S e$ of $78.5 \%$ and $S p$ of $66.5 \%$ were similar to those reported in prior studies. Pilla et al. (2013) reported Se of $73 \cdot 3 \%$ and $S p$ of $73 \cdot 6 \%$. Adjustment of user settings from 1 to 12 would allow user optimization of settings. Sensitivities progressively decreased from $95 \cdot 4 \%$ at setting 1 to $47 \cdot 7 \%$ at setting 12 , while specificities increased from $24 \cdot 2 \%$ at setting 1 to $84 \cdot 5 \%$ at setting 12 (Fig. 2).

\section{Reference}

Gonçalves JL, Lyman RL, Hockett M, Rodriguez R, dos Santos MV \& Anderson KL 2017 Using milk leukocyte differentials for diagnosis of subclinical bovine mastitis. Journal of Dairy Research 84 309-317. Published online 27 June 2017, doi:10.1017/S0022029917000267 